\title{
Ethical considerations during human centred overt and covert research
}

\author{
J. P. van Deventer
}

Department of Informatics, School of Information Technology, Faculty of Engineering, Built Environment and Information Technology, University of Pretoria, Pretoria 0002, South Africa

\begin{abstract}
During the research process, several ethical issues have to be considered in ensuring the safety of the research participants. This is especially true in the case of human centred research designs in which people, not objects are the focus of research. This article reviews several of the issues that researchers should consider before, during and after the planning, implementation and completion of the research process whether it is overt or covert research. The article also functions as a tool that researcher may use in the consideration of the ethical value of their research and research results. The article provides the reader with the opportunity to review their own research related behaviour in ensuring that their research process is ethical in nature, as well as allows for the consideration of the safety and security of the research participant, and final contributing to society as a whole.
\end{abstract}

\section{Introduction}

During the research process, several ethical issues have to be considered in ensuring the safety of the research participants. This is especially true in the case of human centred research designs in which people, not objects are the focus of research. As one may see in Fig. 1, the research process consists of four distinct research elements namely, the process of designing the research also known as developing the research design, implementing the research design, analysing data obtained from 


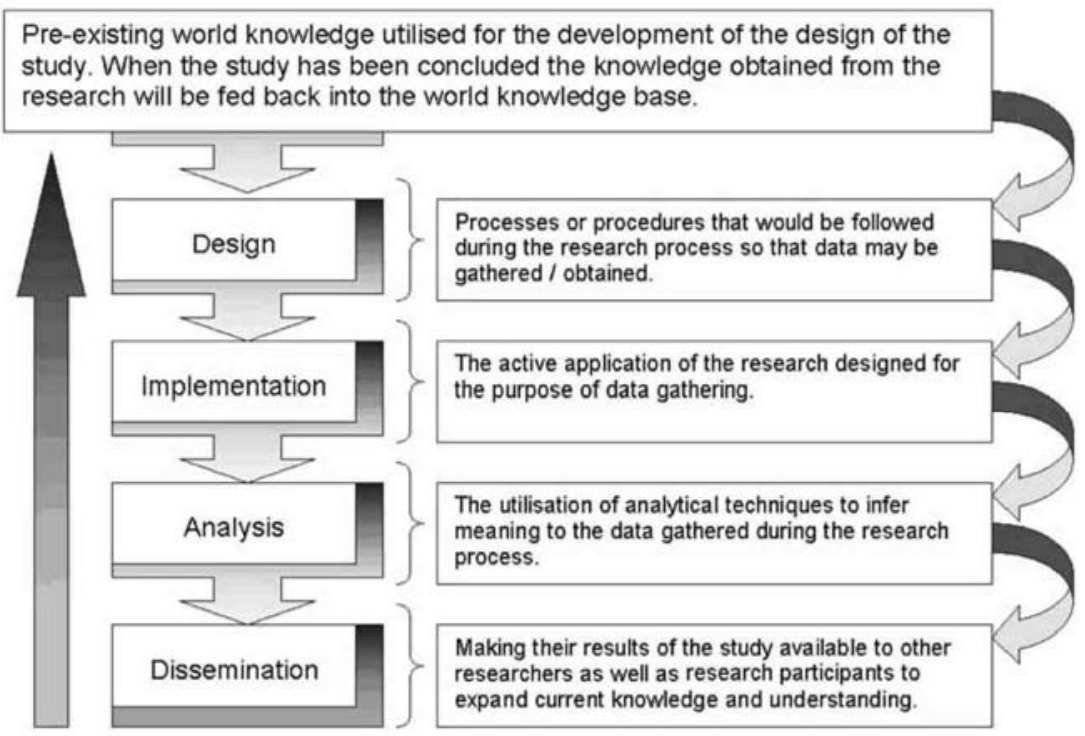

Fig. 1 The elements of a research process (adapted from Mouton 20001)

research activities, and finally disseminating the research results after analysis has been completed (Mouton 2001).

Each of these four elements/steps has a specific set of ethical principles, ideals and/or issues associated with it. In accordance with these research elements the ethical principles/issues during the application of each of the elements of the research process will be discussed. Please note the approach centres on research processes and procedures associated with human-centred research. Animal centred research is slightly different in terms of the application of certain principles. This article focuses exclusively of human-centred research.

According to Phillips (1985) research ethics are the guidelines that are utilised to ensure that, during the complete research process, effective communication to all participants and recipients of research processes and results are ensured, that all research participants are free from harm in all its forms and formats, and to ensure that researchers "actively do good" in society or societal groups. The idea of research "actively doing good" within society most likely associate itself with the avoidance of arbitrary research ending in superfluous results. The final result of research is supposed to be the enhancement of the social good. If research processes cannot achieve this then it might be viewed as wastage of social resources or social capital.

In the following sections of this article, this author will discuss some of the potential ethical considerations during each of the specific steps of the research process as indicated by Mouton (2001). These considerations will allow a researcher to avoid potential wastage of social capital, thereby ensure that research results fulfil the requirement of actively "doing good" within the social sphere. The discussion of research ethics in human related research focussing on overt and covert research is based on the integrated summarisation and interpretation of the APA (1992), Benjafield (1994), Berg (1998), Dane (1990), Kerlinger and Lee (2000), Leedy and 
Ormrod (2001), Mook (2001), Mouton (2001), Newman (1997), the Nuremberg code, Phillips (1985) and Willig's (2001) treatise of the subject of research ethics within chapters of the respective authors and organisations' publications.

\section{Designing the research process}

Ethically, any aspect that has to do with how the design of a research process may influence individuals and groups of people directly and indirectly, should be considered whilst the design or structure of the research process is being developed. Generally research focuses on increasing humanity's knowledge. There is however, a general fear associated with the research process as well as how research results may be utilised after the process has been completed. Though the fear may not be expressed in a tangible form, this fear exists within the subconscious of society. According to the respective authors consulted, Nazi experimentation during the Second World War lead to the development of the Nuremberg code of experimental research ethics to avoid the dangers associated with research as well as potential scientific misconduct. The Nuremberg code indicates that "experimental research should yield results for the good of society and that the results should be unprocurable by any other methods". Experimentation should not be "random" or "unnecessary". Though the Nuremberg code focuses only on experimentation with human subjects, it does provide an insight into the earliest form of research ethics. The implication of the Nuremberg code is that research should not be arbitrary in nature and that the associated research design's sub-sections should not be added arbitrarily. When developing a research design the researchers should weigh the necessity of the research process against any invasiveness into societal privacy.

Subsequently, researchers should maintain an ethical balance to promote intellectual freedom and contribute to the knowledge of society (through fair treatment of research subjects) without endangering societal groups or individuals engaged within the research process. In other words the researcher should justify research in terms of the "risk benefits scale" to avoid danger to the subjects of the research process as well as the recipients of the research results.

Principally, whilst the researcher is engaged in designing a research process, ethical considerations and professional codes of conduct should be utilised to guide the design process to avoid potential harm (in any form or format) that is reasonably avoidable during the research process. This author refers to "reasonably avoidable danger" since a researcher is not a fortuneteller and cannot foresee all the potential dangers associated within any and all research scenarios. All that can be hoped for is that foreseeable danger can be avoided and circumvented to ensure the safety of the research subjects and all associated research participants.

During the development of the research design, researchers should consider that which will be done (methodologically) or how it would be done (method wise) within the research environment. A researcher should decide whether on not to utilise an overt or covert research design. Deciding on either design would influence the way in which the researcher would obtain informed consent as well as how the research would gather data associated with his/her research design. In principle, informed consent refers to a research participant indicating that $\mathrm{s} /$ he was informed of all the aspects associated with the research process and that $\mathrm{s} / \mathrm{he}$ voluntarily participate in the research process, free from any form of coercion, or compulsory participation. 
This is a problem for covert research since the participants weren't informed of the research process being undertaken. The participant will (hopefully) be debriefed after the completion of the research process regarding their "participation".

In overt or visible research designs, the subject of the research process is aware that $\mathrm{s} / \mathrm{he}$ is part of an investigation into a specific phenomenon. Within this specific design the research subject also provides "informed consent" with regards to data collection and research participation. All relevant information associated with the research process should be provided to the research participant. If this cannot be done to ensure the safety of the research participant (keeping the participants free from harm or any type of unnecessary danger), then the research designs should be altered/refined to ensure that valid information for participatory reasons should be made available efficaciously so that research participants would know exactly what they might expect within the research process. Within an overt design a participant may, and should be allowed to withdraw his/her participation if the participant deems the research to encroach into his/her private life (unless of course that is the objective of the study as outlined to research participant and consented to by the participant). In some cases such as in quantitative research designs where questionnaires are utilised, if the respondent completes the questionnaire, then the respondent would have provided "implied consent" since the respondent wouldn't have completed the questionnaire if they did not consent to the utilisation of their data as gathered via the questionnaire. One consideration that has to be taken during the utilisation of questionnaires is that the data gathered from the questions is to be utilised as specified within the questions itself. The data cannot be arbitrarily utilised for whatever purpose the research chooses it to be utilised for. This encroaches on the right of the participant to control his/her personal information as well as consent for the utilisation of said data.

In some cases of overt research designs, the researcher might utilise misdirection to mislead the research participants into believing that the research process focuses on one aspect even though it actually focuses on something else. Though the process is misleading to the research participants (by originally misinforming research participants), after the research process has been completed the research participants are debriefed and informed about the actual purpose of the research process. In such a case the researcher ensures that the research participant does not experience any physical or emotional endangerment as a result of the research process. If the researcher cannot ensure this, then according to Nuremberg code, the research processes was unethical in nature.

Another type of research design with its own specific issues is the covert (hidden) research design where the subjects of the study don't know that they are being studied or involved in a research-based investigation. With the development of such a design the researcher should be absolutely sure that the data obtained from this type of study couldn't have been obtained via any other means. Within covert research a certain level of deception is required in that the identity of the researcher is obscured from the subjects of the study. The subjects or the "research participants" do not know they are being studied or that a research process is imposing on them. Subsequently one cannot refer to the subjects of covert research as participants, seeing that they are not participating, merely reacting or being observed in their "natural environment" depending on the type of research being undertaken. Proponents of covert research argue that if they do not utilise a covert research design then the behaviour of the subjects would change due to interactive factors, thereby influencing the relevance of 
the data gathered during the research process. One example that is cited ad nosium by the proponents of covert research is the Hawthorn experiment resulting in the description of the Hawthorn effect where overt research subjects altered behaviour based on their knowledge that they were being observed.

If the researcher chooses a covert research design, the researchers should be very careful in justifying the research in terms of the risk benefit scale utilised for the purpose of ethical research considerations. Such consideration should not occur arbitrarily-in such a case ethical codes of conduct should be utilised to guide the researcher as well as institutional approval should be sought in ensuring an ethical research design. As stipulated earlier, the principal purpose of research is basically "doing good". The purpose of an institutional revue board would then be in identifying whether or not the final results of the research would enhance society as a whole. If a review board views the research as arbitrary in nature, then the board would recommend that either the research process be reviewed, revised or in some cases, ended. The review board is guided by means of acceptable codes of conduct as well as legal standards to ensure professional conduct and codes of practice.

Covert research is the only type of research where a modicum of deception is allowed. Under normal research circumstances deception would be frowned upon as part of the research process and should be avoided at all cost. If a certain amount of deception is required as part of the measuring scales to obtain relevant data as required within the investigation, then the research design should include a session in which the research participants should be debriefed in terms of the results as well as the type of deception utilised in the research process. After the debriefing session the research participants should be given the choice to allow the utilisation of the gathered data or the omission of the data from the research analysis. If the research participant wishes the omission of the data then the researcher should respect the research participant wishes. This might leave the researcher with insignificant data, however it is one of the dangers that should be considered when utilising deceptive research methods.

While the researcher is involved in designing the research process, the research should also keep in mind that there are several ethical issues in terms of subjects' participation within a research process. A few of these ethical issues include legal jeopardy (when a research participant inadvertently breaks the law due to the stimulation or induction resulting from the research process or when the researcher participates in illegal activities as part of an ethnographic research approach), physical harm that may endanger a research participant (potential physical danger that the research participant may experience), psychological dangers associated with participating in the research process such as for example the potential loss of self-esteem or the traumat-isation of the participant by the research process.

At the end of the day the research design as set out by the researcher should fulfil ethical requirements as indicated by certain ethnically acceptable code of conduct, legal requirements, and requirements of everyday social acceptability. If the researcher does not ensure that his or her research is ethical in nature (in terms of its design and expected outcomes based on the design) then when it is implemented it may lead to what may amount to "professional suicide" within the field in which the researcher practices. It might even have legal ramifications for the researcher in terms of incarceration or fines. Research designs should ensure the safety of all research participants in terms of physical, emotional and psychological aspects. If a research 
design cannot ensure this then the research process has to be redesigned or modified to engage the ethical process.

After a researcher has, within reason, ensured the ethical nature of his/her research design then the researcher has to ensure the ethical implementation of said design within the environment in which the research process will take place. An ethical design with an unethical implementation is equal to an unethical research process. The researcher is obliged to ensure that both the research design and the research implementation (which includes the data gathering process) ensure the overall safety and privacy of the research participants as well of the advancement of society. In the next section the author will discuss the implementation of the research design within an ethical framework.

\section{Implementing the research design}

The process of implementing the research design relates to actually doing the research. First of all the researcher has to gain access to the environment in which the research will be conducted. In a general overt research design this implies that the researcher engages potential research subjects or research participants through an acceptable sampling format. The researcher has to obtain informed consent or implied consent from the identified potential research participants before continuing with the research process. To obtain consent the researcher has to inform the potential research participant of the general research design as well as disclose any potential harm that might endanger research participant whilst engaged in the research process. The research participant should also be informed about processes and procedures involved in participation as well as their right to withdraw from the study at any time or when they choose to do so. A very important aspect of implementation of the research design is ensuring the participant's right to privacy in terms of anonymity and confidentiality. If the research cannot ensure the privacy of a research participant then the implication is that the research design is flawed in nature, and that potential research participants would avoid engaging within the research process and that the relevance of the data obtained within the study would be questionable (due to the potential arbitrary nature of the research).

If a participant consents to engaging or participating in the research process, then the researcher has to ensure that the elements of the research process as described to the research participants is upheld and that the research participant is kept from harm. In other words, the researcher should keep to his/her word in terms of what s/he informed the participant about the design, and utilising the design as described to the participant. In terms of the Nuremberg code, all subjects within a research process should be able to bring the research process they are involved in to an end if they deem the process to endanger their physical and emotional wellbeing, and a researcher should be capable of discontinuing the research process if s/he identifies components (not identified during the planning of the design) that may endanger the research participants. The researcher may not alter the research designed after implementation without the consent of the research participants. This consent should be gained by a full briefing regarding the alteration of the research design and why it is necessary to change the research design. If the research participant wishes to disengage from the research relationship, then the researcher should accept the 
participant's decision in this regard. The researchers should respect the participant's right to protect their personal integrity.

In terms of a covert research design, the implementation process is fraught with ethical dilemmas. Simple reason being that covert research has a component of deception associated with it. The research subjects do not know they are actually engaged in a research process. Fortunately covert research designs are limited in the type of research process associated with it. For example, though not impossible, it is difficult to conduct experimental research without informing subjects that they are actively involved in a research process. A certain amount of covertness may actually be involved in overt experimental research, however in such a case the research participants are well aware that they are involved in a research process. This type of research process is mediated by the researcher and the research demands a certain amount of openness in the example of experimental research. The covertness in this case would be to ensure the integrity of the study as well as the final reliability and the validity of the gathered data. However in such a case the research participant will and should be informed about the covert aspect after the research process has been completed.

In pure covert research the research subject (not the research participant) is unaware of the fact that $\mathrm{s} / \mathrm{he}$ is being observed or studied for research purposes. One might argue that deception is not involved since the research subject is unaware of the study therefore they are not been deceived through the omission of information. This is false. They are being deceived. The deception associated with covert research is in the omission of the research's identity and/or existence within the subject's context/environment. The researcher becomes indivisible within the environment in which the subject related phenomena is being studied. This creates serious questions regarding the right to the individual's privacy, anonymity and the confidentiality of the research subject. The researcher is principally intruding into the private life space of the research subject and it may be equated to surveillance or spying upon the research subject-this relates to the development of a research panopticon (subjects are being observed without their knowledge). This also creates a situation where a research subject cannot withdraw from a research process since basically the research subject does not know that $\mathrm{s} /$ he is involved in a research process. It also creates another ethical dilemma for the research in that the researcher may get to known too much about the research subject. For example, in the case of using observation as a research process the subject of the study may be juvenile delinquents. In this study the researcher may observe criminal behaviour. The researcher now experiences a condition of legal dualism — does the researcher reports observing criminal behaviour thereby revealing the research process, or does the researcher remain silent theoretically "promoting" criminal behaviour? By not reporting the criminal act it may be argued that the researcher is supporting the escalation of the delinquent behaviour through the promotion of criminal punishment avoidance.

In covert research the subject's self-determination in terms of the right to privacy and the right to withdraw from the study is nonexistent. Researchers may delude themselves into believing that they have adequate safeguards in terms of protecting the identity of the research subjects, however, who protects the research subjects from the research's intrusion? With a covert research design questions of confidentiality and the privacy of the research subjects is a very sensitive issue. Who protect the subject? Who ensures the safety of the subject? Who protect the well-being and self-esteem of the subject? In such cases the research may answer by indicating that 
institutional review boards and ethical codes of conduct guide the research process, however the question still remains, who gives the researcher the basic right to investigate a research subject without the research subjects consent? In such a case a covert research design finds itself on a very slippery slope with regards to ethical research considerations. Covert research should only be conducted in very specific situations where for example, the behaviour of the research subject would intrinsically change if the subjects knew that they were being investigated or that they where being involved in a research process. Another issue to consider with regard to covert research is the safety of the researcher. Will the research be safe? The question of safety refers to safety from legal recourse as pursued by the research subjects, public protectors and/or professional associations. How will the researcher ensure his/her own personal physical and legal safety in for example an ethnographic study of a "vampire sect"? The author would like to keep this question open to interpretation and debate.

In many cases the implementation of a specific research design quickly resolve potential ethical issues associated with a specific research process. In such case, the researcher would eventually discover, hopefully at the beginning of the research process' planning, design and implementation phase, that the research would eventually lead to unusable results. The researcher would then be capable of discontinuing the research design in favour of reviewing the research process, resulting in a more efficacious research design. The lessons learned as well as the amount of research data gathered in the preliminary implementation and evaluation of the research process would then be utilised for the purpose of refining and reviewing the research design. The review and refining process associated with preliminary implementation of the research design, allows the research to ensure that the eventual research processes is as efficacious as possible in the gathering of relevant data. When a researcher starts reviewing the research design late in the process, then the researcher has basically been wasting time and resources on a poorly constructed design that would yield unusable and arbitrary results. This result in an ethical question with regard to the utilisation of the respondent's or research subject's time and personal resources since the respondent's personal time is a resource belonging to the respondent. When this happens the implication is that the various aspects of the research design was not efficaciously considered and reviewed before the implementation of the research process.

In conclusion when a research design is being implemented and utilised for data gathering purposes, then the needs and requirements of the research subject has to continuously be taken into consideration. This is done for the simple reason that the research participants are giving their time and experience to the researcher within the designed process. The safety of research participants should always be taken into consideration. If a researcher does not do this, the implication is that his/her research is being conducted in an unethical atmosphere.

\section{Analysing data obtained from research}

After the research process has been completed in terms of data gathering, then the research data has to be analysed for the purpose of drawing inference from it. Whilst analysing data, the researcher has to take certain aspects into consideration. One of thisisfor example the objectivity and integrity ofthe analytical process and individuals 
assisting in analysis. Certain research methods indicate that the researcher should utilise personal experience with regard to research process, however the researcher should avoid the utilisation of these personal experiences to such an extent that it clouds the researcher's analytical capability. Though researchers should acknowledge how personal perception influenced the gathering as well as analytical process associated with the research gathering techniques and interpretation of the data associated with the research process, the researchers should remain objective in terms of not interpreting data to indicate what the research feels it should indicate. In other words, the researcher should avoid the construction of creative results. If a researcher does this during the analytical process, it may be interpreted as professional fraud also known as research fraud that may be defined as the fabrication and/or falsification of research results.

Preferably a researcher should also analyse the data gathered through the utilisation of acceptable analytical techniques. However if a researcher uses any technique that may be unfamiliar or wholly new, then the researcher should document the analytical process thoroughly. This is done so that the research results may be "reproduced" by the utilisation of exactly the same research process or analytical technique to ensure for reliability. It also allows the research fraternity to review and evaluate the veracity of the potentially innovative technique. The documentation of the technique will also allow other researcher to analyse the results and process via standard processes to ensure that the results obtained was not obtained via data manipulation. This eventually ensures the reliability and the validity of the research results. If a researcher utilises an obscure analytical technique that has not been thoroughly documented, then the research results may be drawn into question. In some cases researchers utilise obscure analysis to mask impoverished techniques in terms of data gathering and data analysis. It implies that the research infers analytical results that are not indicated by data.

The above-indicated section refers to the distortion of data and the manipulation of resources for the purpose of analysis. Data distortion refers to the distortion of methods of data gathering, the distortion of methods for analytical purposes, and the distortion and misinterpretation (misconduct) of the scientific responsibility associated with the enhancement of society. This may most likely occurs due to the pressure that is placed upon researchers to produce results. However researchers should weigh the pressure placed upon them against the validity of the results in enhancing society as a whole. If results were distorted then the implication is the action society takes related to the distorted data/results would itself basically be an extension of this distortion, and in the most cases, counter-productive. An example would be that if research indicated that smoking is good for a person's health, one would have a global smoking craze since research indicated that smoking is good for you. This could happen due to society's "trust" in scientific research techniques and results being truthful and potentially dependable. However if your research indicates that the previous research associated with smoking was falsified, then the public could lose trust in the scientific community. Basically this may be equated to scientists "crying wolf".

The falsification and the misinterpretation of data is a very critical issue in the research community. This usually happens when a researcher has completed a research process, has all the data, and now feels as though s/he has to say something about the results. Is the research is inconclusive, then the researcher should indicated the result was inconclusive. 
There's also an issue related to the sharing of data gathered during the research process. Should a researcher share his data with other researchers? Personally, this author thinks that if the data has been anonamised sufficiently, and all traces of the research subjects' identity has been removed, then there is no specific ethical issues associated with it. Theprimary purposeofresearchistopromote society, andifanother researcher can provide unique insight into the data that the original researcher could not have provided, then the primary purpose of the research process will have been fulfilled and society would be served. Though potentially useful to share one's data, it should be reviewed in terms of the ethical consideration of accuracy. For whom would the data be accurate? Would it be accurate only for the researcher (who gathered the data) or would it also be accurate for the recipient of the data (who was removed from the research context)? One should consider that the data recipient may not necessarily have the background associated with the obtained results in mind when s/he analyses the results. In terms of promoting objectivity in analysis, this would have a positive effect, however it could also have a negative effect on the analytical completeness required in rigorous research. The researcher should consider whether s/he is willing to exclude relevant experience (which cannot in essence, be effectively documented) during the transfer of results to another researcher.

The above-indicated discussion refers to analytical procedural integrity. In principle it implies that a researcher may not distort or manipulated the data in such a way as to produce results that the researcher believes the data should provide. The results of research should speak for itself through correct interpretation and analysis. It may not be distorted to service the ego and professional pride of the researcher. It this occurs, then the researcher breaches the trust society places in research to produce adequate and social enhancing knowledge to be utilised for social growth. In the following section ethical issues associated with the dissemination of research results will be discussed.

\section{Disseminating the research results}

After the completion of the research process and the associated analysis of data, the researcher has to disseminate research results to all parties associated with the research process to ensure the completion of the research process, as well as the enhancement of the "knowledge-base" from which the information utilised for the formation of the study originated. During the 'sharing' or rather, dissemination of the research results, the researcher should consider the privacy of the research participant. It is very critical during the process of result dissemination that any information pertaining to any participant should be utilised in such a fashion as to avoid the identification of said research participants.

Particular to the consideration of information omission for the protection of privacy, is whether or not a research participant would be willing that the researcher should be allowed to utilise his/her data for dissemination purposes. If the research participant doesn't like the idea of disseminating potential information to other individuals, of for example fears identification in the case of sensitive research foci, then the researcher should still allow the participant the right to request omission of results from the analytical side of the study. It may sound as thought the protection of privacy cannot be so far fetched, however the researcher should at all times during the 
research process be willing to protect the participant, and the dissemination of the results is after all part of the research process.

Thought it might influence the results of the study, the researcher should still respect the participant's will in this regard. If this is not done, it may be seen as "poisoning the well”. If the researcher doesn't respect the wishes of the participant, the participant might in effect communicate his/her dissatisfaction to other individual, leading to individuals in his/her direct community to avoid other researchers in the future. Professionally, other researchers might frown on this type of action since it appears as though the researcher uses the participant without providing them with sufficient feedback or "control” over their personally provided information that is part of their personal field of experience. Technically this may also place the researcher in legal jeopardy. The informed consent forms does not indemnify a researcher from legal action, and subsequently if a researcher doesn't respect the wishes of the associated research participant, then the participant may in effect place a legal claim against the researcher. The informed consent form as well as the privacy stipulations in all forms utilised to communicate with the research participant may actually be utilised in support of such a legal action against the researcher, as it is a "good faith" agreement between the researcher as well as the research participant. This agreement is legally actionable if the participant wishes to withdraw his/her personal information from the process and the researcher does not comply with the good faith nature of the consent and other information forms.

It is the responsibility of the researcher to honour his/her agreement with the associated research participants, and if they wish it, to retract or remove the participants results from the information to be disseminated to the research community as well as all relevant parties.

Another significant ethical responsibility during the research process is the ethical responsibility held by a researcher in terms of other researchers. All information utilised that originates from other sources should receive due accreditation, basically "giving credit to where credit is due". Duplicate publication of results should also be avoided unless of course when the publication differs significantly in terms of the analytical process associated with the utilisation of the data. For example, a researcher could have collected statistical results associated with deviant behaviour trends, and then disseminate the information through one channel, however, the statistical result may then be utilised for example to explore new statistical techniques of analysis. The research user/reader may then, in this specific example, utilise the one channel to evaluate the content reported in the other channel, basically utilise the formal analysis of the results to evaluate the secondary exploration of the new statistical technique. When a researcher does this, s/he should make sure that the utilisation of the research results for secondary purposes would not expose the research participants in any form or format, as well as that the secondary analytical technique of analysis was mentioned in the consent forms. In some cases the researcher may utilise the data as presented in his/her publication for secondary analysis without consent forms, since the data utilised has already been communicated to the research community and the representation of the results has already been 'anonamised' to ensure the privacy of the research participant. The results as communicated is utilised, not the original data that has been utilised for specific purposes such as the investigation of a specific social phenomenon. In such a case the researcher would utilise already communicated results for other analytical purposes. 
Before the result of the research is "added to the human knowledge-base", the researcher should have the results reviewed by other researcher to ensure that the analytical conclusions are 'correct' and unbiased. Research reviewing allows the researcher to avoid things like unintentional errors that may later be interpreted as research fraud or plagiarism. During an extended research process, the researcher might, due to human nature, "forget" to include some of the required aspects within his/her research report for example to acknowledge other individuals intellectual property, that will be utilised for dissemination purposes. Researchers are after all also only human. However, if a researcher allows other researchers to evaluate the results before the results are disseminated, then the researcher may avoid potential research fraud or plagiarism allegations by allowing other to identify potential problems, and then rectify the identified problems through self-review of the final research report. Review by other researcher will also allow researchers to ensure that their conclusions are fair and unbiased. The interpretation of the results should be critically reviewed to ensure that all possible avenues in interpretation have been covered to ensure complete and thorough exploration of the results.

The report should also be provided for evaluative purposes to ensure that professional misconduct can be avoided. If a research report is utilised without evaluation, then the research community might question the validity, reliability as well as the professionalism of the research conducted for the production of the report. In the long run it would be ethically advantages to allow evaluation of ones anonamised research results.

In terms of covert research the research should undergo strenuous evaluation before the results may be disseminated and/or made available to any person or group not restrained by research ethics. The reason being naturally that the subjects involved in the study may still not know that they where part of a specific study. This would influence the way in which the research results would be disseminated. If the research results are too specific, then the individual "research subjects" may be identified and in some cases, stigmatised. This is something that most researchers would like to avoid under any and all circumstances. Professional review will only ensure that all traces of identifying matter have been removed and that the identities, including the geographical area of the study has be removed or obscured from the reader/recipient of the research results.

\section{Conclusion}

In Conclusion, ethical considerations during the research process is not only professionally required to protect the rights of the research participants, it is also necessary to ensure the efficacious development of utilisable "knowledge" that will enhance the collective knowledgebase of humanity in total. If researchers conduct their research in an unethical manner, not only will the research process become stigmatised in terms of its conduct towards research participants, but it will also influence the development of the collective knowledge that humanity possesses. People will not want to be associated with research in an unethical or damaging environment and subsequently research participants would be few and far between. This would lead to an underdevelopment in human knowledge, leading to a slowing of development, and even possibly, in the most extreme scenario, the stagnation of human development in total. 


\section{References}

APA.: Ethical principles of Psychologists and code of conduct. Am. Psychologist. 47, 1608-1610 (1992) Benjafield, J.G.: Thinking Critically about Research Methods. Allyn and Bacon, Boston (1994) Berg, B.L.: Qualitative Research Methods for Social Sciences, 3rd edn. Allyn and Bacon, Boston

(1998) Dane, F.C.: Research Methods. Brooks/Cole. Pacific grove, California (1990) Kerlinger, F.N.,

Lee, H.B.: Foundations of Behavioral Research, 4th edn. Harcourt College Publishers,

Fort Worth (2000) Leedy, P.D., Ormrod, J.E.: Practical Research: Planning and Design, 7th edn.

Merrill Prentice Hall,

New Jersey (2001) Mook, D.G.: Psychological Research: The Ideas Behind the Methods. Norton,

London (2001) Mouton, J.: How to Succeed in your Master's and Doctoral studies. A South African Guide and

Resource Book. Van Schaik Publishers, Pretoria (2001) Newman, L.: Social Research Methods. Qualitative and Quantitative Approaches, 3rd edn. Allyn and

Bacon, Boston (1997) Nuremberg code. Available [Online]: http://ohsr.od.nih.gov/nuremberg.php3

Phillips, B.: Sociological Research Methods: An Introduction, Chapter 16, pp. 524-548. The Dorsey

Press (1985) Willig, C.: Introducing Qualitative Research in Psychology. Adventures in Theory and

Method. Open

University Press, Buckingham (2001) 